\title{
Portable Health Clinic as a Telemedicine System with Appropriate Technologies for Unreached Communities
}

\author{
Rafiqul ISLAM-MARUF ${ }^{\mathrm{a}, 1}$ Ashir AHMED ${ }^{\mathrm{b}}$ Fumihiko YOKOTA ${ }^{\mathrm{c}}$ Kimiyo KIKUCHI $^{\mathrm{d}}$ \\ Mariko NISHIKITANI ${ }^{\mathrm{a}}$ Rieko IZUKURA ${ }^{\mathrm{a}}$ Yoko SATO $^{\mathrm{d}}$ \\ Yasunobu NOHARA ${ }^{\mathrm{e}}$ and Naoki NAKASHIM ${ }^{\mathrm{a}}$ \\ ${ }^{a}$ Medical Information Center, Kyushu University Hospital, Japan \\ ${ }^{\mathrm{b}}$ Graduate School of Information Science \& Electrical Engineering, Kyushu \\ University, Japan \\ ${ }^{\mathrm{c}}$ Institute of Decision Science for a Sustainable Society, Kyushu University, Japan \\ ${ }^{\mathrm{d}}$ Dept. of Health Sciences, Faculty of Medical Sciences, Kyushu University, Japan \\ ${ }^{\mathrm{e}}$ Faculty of Advance Science and Technology, Kumamoto University, Japan
}

\begin{abstract}
Poor healthcare infrastructure is the main barrier for providing quality healthcare services to rural communities in developing countries. Thus, these populations remain unreached, and there is a need to establish a method for ensuring the provision of appropriate and adequate healthcare services to these individuals. The portable health clinic (PHC) system has been developed as an effective telemedicine system to meet this objective. A trained village health worker can use this simple system for collecting vital information of the patient, upload the data to the online server, and connect village patients with a remote doctor to enable the provision of online consultancy using video conferencing. Although the PHC was initially developed to ensure primary healthcare service with a focus on noncommunicable diseases, a major cause of death, gradually, tele-pathology, tele-eye care, maternal and child health care, and COVID-19 care modules have been added to provide special treatment in these areas as per local needs. The modular PHC system will continue to grow with the addition of novel features that aim to address the local needs. The low-cost and easy operation of the PHC system make it ideal for ensuring global health coverage in communities where inadequate medical facilities and poor-quality healthcare resources remain major issues.
\end{abstract}

Keywords. Telemedicine, primary healthcare, unreached communities, home delivery healthcare service, triage, patient engagement, population management

\section{Introduction}

Although healthcare service is a basic right of every human being, most individuals who live in rural areas of developing countries are deprived of this service. The main reason for this is the lack of adequate and appropriate healthcare facilities and resources, including doctors, nurses, and medical assistants. Several initiatives have been

\footnotetext{
${ }^{1}$ Corresponding Author, Rafiqul Islam Maruf, Associate Professor, Medical Information Center, Kyushu University Hospital, 3-1-1 Maidashi, Higashi-Ku, Fukuoka 812-8582, Japan; E-mail: rimaruf@med.kyushuu.ac.jp
} 
undertaken for improving the existing healthcare facilities worldwide [1-3]. However, for unreached communities of developing countries, there is a need for technically simple, usable, sustainable, and inexpensive healthcare service systems. The portable health clinic (PHC) system has been developed with this target in mind in Bangladesh under the joint collaboration of the Kyushu University of Japan and Grameen Communications of Bangladesh [4-5]. This system can serve as a model for developing countries because the resource availability and conditions in most such countries are similar to those in Bangladesh.

As shown in Figure 1 (left-hand image), the basic model of the PHC system includes the following four major components: (1) a set of medical sensors to be used for collecting patients' health data, (2) an online server system for data storage and sharing with remote doctors, (3) remote doctors for providing telemedicine service, and (4) a health worker (paramedics with 1-3 years diploma certificate) who collects the patients' health data and coordinate with remote doctors for tele-consultancy when required.
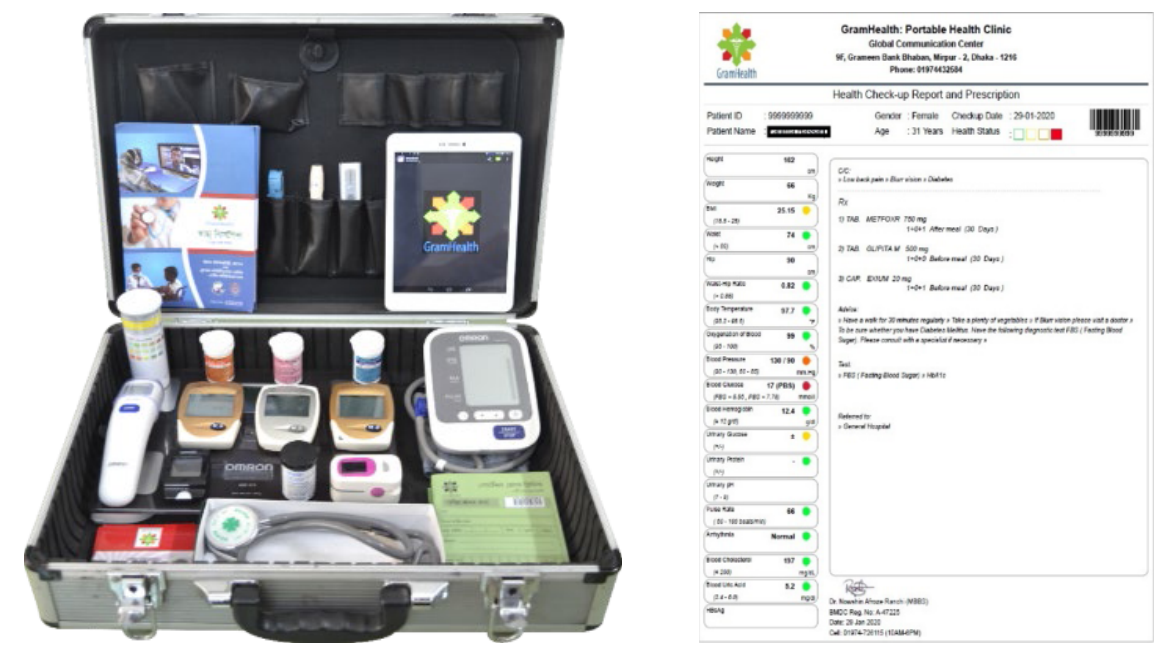

Figure 1. The 'portable health clinic' system box and prescription

In the case of service delivery mechanism, the PHC system has adopted the philosophy that the clients are the most important factor that contributes to the sustainability of any business and the service provider should go to the client with the service as a sign of respect. Therefore, the PHC system has been designed to deliver healthcare service at the doorsteps of the rural community members to ensure easy accessibility of the service for the beneficiaries. So, the health clinic needs to go to the patients' homes instead of asking the patients to visit the clinic. Thus, the present novel system has been named 'the portable health clinic,' and the health worker provides homebased healthcare service to a patient by visiting the patient's home with the PHC system [6-8].

Before designing the content of this system, we studied the disease pattern in Bangladesh and found that $70 \%$ of the deaths in Bangladesh were attributable to noncommunicable diseases (NCDs), such as hypertension and diabetes. Thus, the PHC system has been designed to provide primary healthcare with a special focus on NCDs. While delivering the service in the unreached rural communities, it was found that 
primary care is not enough and, in several cases, advanced patient care is necessary. Then, a new module of tele-pathology has been added to the PHC system as described below to support the remote doctor with further detailed patient reports. This additional feature has highly empowered remote doctors in the provision of secondary level of treatments via telemedicine. The remote doctors do need to refer the patients in some cases to the nearest district hospital when it is difficult to administer and support treatment via the tele-health system. This referral system forms a crucial part of the PHC system wherein only patients with serious conditions and specific needs are referred to sub-district or district hospitals that have limited resources. This enables the referral hospital to ensure optimal use of their limited healthcare resources for selected patients who need special care and prevents the unnecessary crowding by low-risk patients. The low-risk patients can be supported by the remote doctors by the PHC service.

The triage feature of the PHC system also plays a crucial role in reducing the workload of the remote doctors. The PHC triage classifies the patients in the following four categories based on their collected health data: (1) green or healthy, (2) yellow or suspicious, (3) orange or affected, and (4) red or emergent. The green and yellow patients are usually supported by the health workers who do not need any medication but receive advice-based preventive care. The remote doctors are consulted for the orange and red patients as well as some selected yellow patients. Thus, the limited human resources of the healthcare system are managed to ensure their optimal use. This is a very important factor for ensuring better healthcare services with the use of limited resources in unreached communities.

\section{The PHC System Operation}

The primary aim of the PHC system is to make primary healthcare services accessible to unreached rural communities. However, the care of patients with NCDs, such as hypertension and diabetes, was considered from the beginning. Thus, the PHC system box includes the following sensors (Table 1):

Table 1. Medical sensors and materials with measurement items in the PHC system

\begin{tabular}{cll}
\hline No. & Device Name & Measurement Items \\
\hline 1 & Thermometer (Omron) & Body temperature \\
\hline 2 & Digital BP Machine (Omron) & Blood pressure and arrhythmia \\
\hline 3 & PulseOxi Meter & Pulse rate and oxygenation of the blood \\
\hline 4 & EasyMate G-Hb & Blood glucose (random) \\
\hline 5 & Manual Measurement Tape & Height \\
\hline 6 & Weighing Machine & Weight \\
\hline 7 & Digital Measurement Tape & Hip, waist, and hip/waist ratio \\
\hline 8 & EasyMate G-Hb & Hemoglobin \\
\hline 9 & EasyMate GCU & Uric acid \\
\hline 10 & Uriesu -Tc (Terumo) & Urinary protein, urinary sugar, and urobilinogen \\
\hline 11 & EasyMate GCU & Cholesterol \\
\hline 12 & Blood Grouping Reagent & Blood group \\
\hline
\end{tabular}

This system box also includes an internet-enabled tablet personal computer, with PHC apps installed for storing health data to the online server. This is accessible to the remote doctor and enables video conferences between the patient and the remote doctor for tele-consultancy. Additionally, it includes a portable printer for printing prescriptions and giving them to the patients on the spot. 
Generally, one health worker provides service in one rural community as an entrepreneur. The health worker visits each registered patient as per the schedule and provides home-based healthcare service. Figure 2 depicts the service process. First, the health worker interviews the patient to collect his/her medical history and then collects the necessary vital information using the PHC service box equipment (1). All data are then entered into the PHC apps, and the patient is classified as per the triage (3). If the patient is classified as green or yellow, the health worker uploads the data to the online server, provides a printed health report, and gives the necessary advice to the patient (7). However, if the patient has any complication that would require medication, or if the triage result is orange or red, the health worker uploads the data to the online server (4), immediately notifies the online doctor, provides the patient's registration identification number to enable data monitoring, and connects the patient to the doctor for a video consultation. Then, the remote doctor prepares a prescription and uploads it on the online server under the patient's profile (6). Finally, the health worker downloads the prescription, prints it, and shares it with the patient (7).
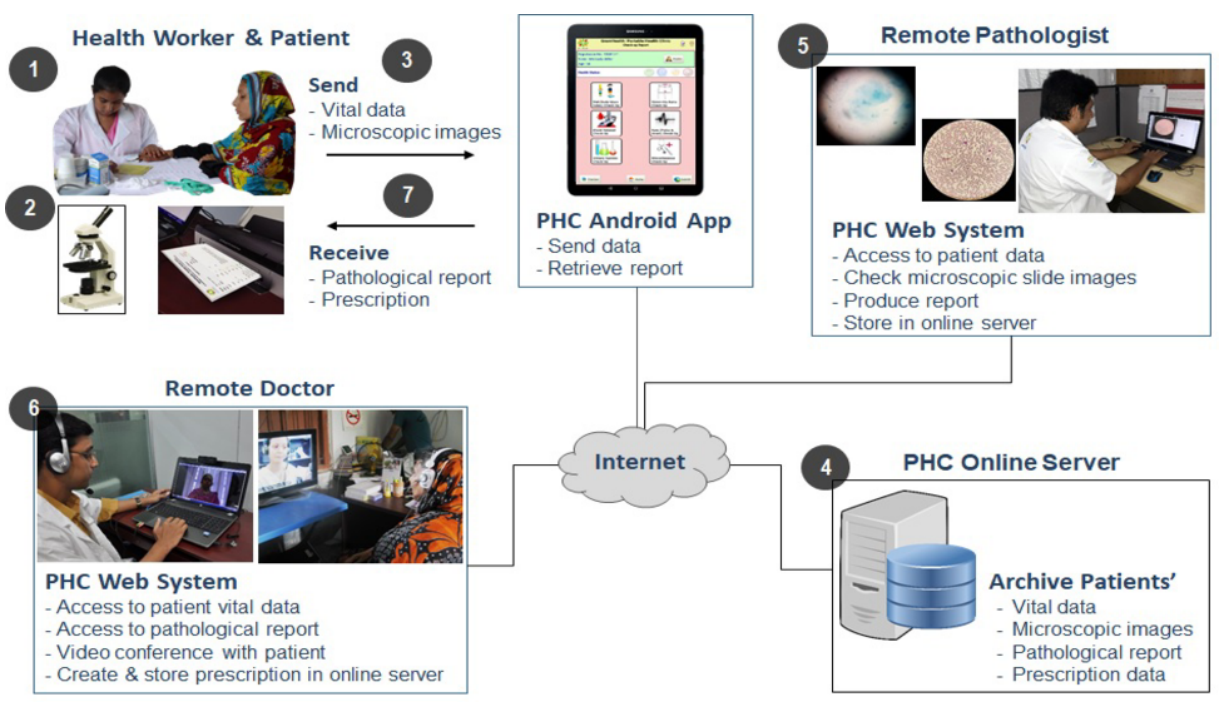

Figure 2. Operation model of the portable health clinic system

In cases where the remote doctor requires additional pathological reports for the patient, the trained tele-pathology staff (a trained rural laboratory technologist) collects a sample, prepares a physical report, and produces a sample slide with 6-10 microscopic images (2). These microscopic images are uploaded to the online server with the physical report via the PHC apps (3). Then, the remote pathologist prepares the pathological report investigating the microscopic images of the slide and the physical report from the laboratory technologist (5). This report is then uploaded to the online server (4). Finally, the remote doctor prepares the prescription with the reference of the pathological report.

The health worker prints the prescription and explains it to the patient before transferring for better understanding. The PHC prescription contains the basic health checkup report and details of the doctor's consultation [Figure 1, right-hand image]. All vital data are marked with color as per the triage result. This is crucial for rural patients 
because they do not understand the significance of the numerical data but can easily understand the severity of the status of each item via color coding of green, yellow, orange, and red. This encourages the patients to follow the advice of the doctors and/or health workers.

\section{Personal Health Record (PHR)}

Today, PHR is considered an important tool for healthcare services. This is more important in the case of tele-medication because the doctor cannot see the patient in person. The PHC system creates the PHR of every patient automatically. A patient can monitor his/her own health data with a graphical representation using PHC apps and be aware of their health status. If needed, they can contact the PHC staff with their feedback or complaints. Thus, this basic 'patient engagement' mechanism helps in building the awareness of the patient and contributes to improving healthcare service in the form of 'patient-reported outcome' [9-10].

For data interoperability, it is very important to maintain a standard PHR structure. Currently, there is no available international PHR standard. The PHR in the PHC system has been following the Japanese PHR standard although it does not maintain all 42 items at this stage [11]. Gradually, with the development of the PHC system, the remaining items will be added to the system.

\section{Special Features}

The rural communities of developing countries experience various diseases, similar to other populations. Thus, when the PHC program offers primary healthcare, sometimes people are dissatisfied because they also require the next level of treatment for their diseases. Therefore, the PHC system has added new modules with advanced features that would serve these higher needs of the community. So far the PHC system has added (1) an NCD module in the basic service, (2) a tele-pathology module, (3) a tele-eye care module, (4) a maternal and child health $(\mathrm{MCH})$ care module, and (5) a COVID-19 care module [12].

\subsection{Non-Communicable Disease}

Seventy percent of all deaths in Bangladesh are attributable to NCDs. Thus, when the basic module was designed, a special focus was on two major NCDs, hypertension and diabetes [5]. Thus, the medical sensors for blood pressure measurement and glucometer have been added to the sensor sets. The PHC system has also adopted the triage concept of NCDs of Japan, with modifications as per the Bangladesh context.

\subsection{Tele-Pathology Service}

As a telemedicine service system, the PHC system offers service assisted by remote doctors. These doctors are unable to see the patients in person; therefore, they need to obtain as much patient information as possible. The PHC system box contains limited sensors for measuring only the basic vital information. In many cases, remote doctors 
require some pathological data. Unfortunately, there is a considerable shortage of pathologists and pathological laboratories in rural Bangladesh. However, there are many rural pathology laboratories operated by laboratory technologists with 3 year Diplomas in pathology. The tele-pathology module of the PHC has empowered these laboratory technologists (local pathologists) by connecting them with urban pathologists. Here, the trained laboratory technologists of the PHC system collect the sample, prepare a physical report, and share the microscopic images of the glass slides of the sample with the urban pathologist [13]. Finally, the pathologist prepares the final pathology report that is used by the remote doctor for their consultancy.

The current tele-pathology service of the PHC system supports the preparation of various reports including (i) hematological report, (ii) biochemistry report, (iii) microbiology report, and (iv) routine examination report of urine, sputum, and stool.

\subsection{Tele-Eye Care Service}

Patients in rural areas of developing countries, such as Bangladesh, are at a high risk of ophthalmic diseases. By contrast, there are only 1,000 ophthalmologists for a population of $160,000,000$, resulting in only 0.063 doctors per 10,000 people. Thus, there is a huge need for ophthalmic support with the PHC system in rural communities. To address this issue, the PHC system has added the tele-eye care module that works on a mechanism similar to that used for the tele-pathology feature [6]. Here, the local trained health worker collects patient data, including retinal images using a digital fundus camera that are shared with the remote ophthalmologist. Finally, the ophthalmologist prepares the report after a video conference with the patients. Presently, the PHC tele-eye care system offers services including; (i) primary investigation, (ii) vision test, (iii) final examination, (iv) refraction, (v) glass prescription, and (vi) eye prescription.

The PHC tele-eye care module aims to adopt the new artificial intelligence-based automated detection algorithm to automatically identify diabetic retinopathy. This technical assistance is expected to contribute considerably toward reducing the workload of local health workers and remote ophthalmologists.

\subsection{Maternal and Child Health Care Service}

There is an urgent need for a home-based delivery service of $\mathrm{MCH}$ care in rural areas because, in many cases, village women have some cultural and religious reservations about visiting distant city hospitals. Furthermore, in most cases, pregnant women from rural areas prefer to consult a female doctor in person. Thus, the MCH care module has been added to provide better healthcare services to rural women and newborn babies [14]. Therefore, a PHC service box has added a handy fetal Doppler machine for checking the baby's heartbeat and a digital ultrasonography machine for monitoring the baby's position in the mother's womb. It follows the guidelines of the World Health Organization (WHO) for monitoring the health of pregnant women and newborns from the beginning of their pregnancy up to six weeks after childbirth [15]. A total of eight checkups are performed during this period by the PHC rural health workers followed by tele-consultancy by a gynecologist in the city.

Additionally, the $\mathrm{MCH}$ service supports village women, especially all women in the reproductive age group (15-49 years) and performs basic health checkups using PHC as preventive healthcare services. 


\subsection{COVID-19 Care Service}

This is the most recently added module to the PHC system. During the COVID-19 pandemic in 2020. This system has a triage function to accurately classify the suspected patients who are recommended for a PCR test [16]. This is believed to lower the testing cost and workload on the test center with limited resources while ensuring that the patients who require a test are subjected to the appropriate examination.

Based on the test results, the system classifies the subjects into the following five categories: green, light yellow, yellow, orange, and red. Green indicates that the patient is healthy and safe from COVID-19 infection. Light yellow means that the patient is slightly likely to have an infection and should maintain home quarantine with selfmonitoring. Yellow shows that the patient is considered probable to have an infection and should maintain home quarantine under the supervision of a PHC health worker. Orange shows that the patient is suspected to have a COVID-19 infection and should immediately talk to a PHC call-center doctor and undergo a PCR test if advised. Otherwise, the patient should maintain home quarantine under the supervision of a PHC health worker. Red means that the patient is very likely to be infected and should undergo a PCR test or visit a COVID-19 care hospital immediately for further follow-up.

The PHC for COVID-19 system can also be used for the remote (or social distancing-based) treatment and follow-up of patients who are home quarantined for suspected or mild-positive COVID-19 infection. The COVID-19 system box contains four items of self-checking medical equipment; one thermometer, one pulse oximeter, one BP machine, and one glucometer. This COVID-19 system and tablet PC allows for the patient's health monitoring, and communication between the patient and doctor for tele-consultancy, while maintaining social distance. This ensures the safety of both the patients and the health professionals, which is crucial for highly infectious diseases, such as COVID-19. This system box can be rented by an individual COVID-19 positive patient or shared by several patients in a community with the support of the local health worker. This system can be easily operated by an individual patient with the aid of the operational manual after a short operational training.

\section{PHC Service Statistics in Bangladesh}

The PHC system started basic healthcare service in 2010. Since then, about 44,500 patients have been managed by approximately 40 PHC systems boxes across 18 districts of Bangladesh until November 30,2020. For these services, 4,500 pathology reports have been provided by the tele-pathology service for 1,900 patients as recommended by doctors.

The tele-eye care service was started in late 2017 in three districts of Bangladesh and have treated approximately 3,000 patients. The $\mathrm{MCH}$ care service was started in 2019 as a pilot program in a district of Bangladesh and has served around 800 women and babies, including 110 pregnant women. As a continuum care of $\mathrm{MCH}$, this service will be continued for all pregnant women, new mothers and babies until 2 years after childbirth.

PHC for the COVID-19 care module was added in June 2020 during the COVID-19 pandemic. As of writing this report, this system had provided care to approximately 1,100 people in three districts of Bangladesh for the identification of suspected COVID19 and provided support for home quarantined probable/suspected COVID-19 patients. 
This is expected to exert a considerable positive impact in terms of reduced workload on the PCR test centers and availability of the test for individuals who really need these services.

This project conducted several surveys to obtain patient feedback. Mostly the village women liked this service because they could receive healthcare services in their home. Although the village people can get primary care service from the public hospital free of cost, they prefer the PHC service because they can avail the service maintaining privacy with an affordable price and avoid long distance travel for visiting nearest public hospitals in the sub-districts. Also, the village women are comfortable with the PHC service because most of the health workers are women and they feel easy to discuss their problems with them.

\section{Discussion}

\subsection{Target Patients}

The PHC system is not only a tele-health system for treating current patients but also a tool for community-based healthcare in the population-management approach. In addition to providing telemedicine services to the orange and red category patients who need immediate medication, the PHC system can provide preventive care to those characterized into the green and yellow categories. As per this concept, developing countries can skip the very long and expensive process of medical service development that has been observed in the past, and achieve modern medical services at a low-cost.

Although the PHC system has been developed for the rural communities that do not have access to adequate and appropriate healthcare, it is suitable and very useful as a home-based healthcare delivery service for elderly patients in urban areas. Most elderly patients have NCDs and need regular checkups and follow-ups. The PHC service could help them avoid regular hospital visits and provide the required healthcare support at home at a pre-scheduled, convenient time. Thus, they can avoid the physical stress involved in traveling to the hospitals and waiting in the long service queues. So far, the urban model of the PHC system in Bangladesh has provided services to 36 apartments in six different areas of Dhaka city covering 1,717 elderly patients. The issue of elderly patients is more prominent in developed countries. Recently, the demand for telemedicine-based healthcare service delivery is increasing, and many developed countries are showing their interest in PHC-type services for their elderly citizens.

\subsection{Technical Challenges}

The PHC system needs to use a number of small, lightweight and low-cost medical sensors for its usability and sustainability. However, only few such high-quality sensors are commercially available. The rest are collected from the available items in the local market. Although the PHC research team evaluates the products from different manufacturers and selects the best possible for the PHC system, there remains a concern of data accuracy. Moreover, there is a need for additional sensors to respond to patients' needs; however, these are not available. The PHC system aims to offer services at a lowcost; therefore, we expect manufacturers to design new, low-cost and portable devices in the future. 
One of the main issues of the tele-health system is that of errors made during data entry and measurement [17-18]. A wrong entry may lead to an inaccurate diagnosis and disallow accurate consultation and treatment by the remote doctor. Better training on system operation of health workers can reduce this error but cannot ensure $100 \%$ accuracy. Alternatively, an RFID-based wireless system can be used to directly transfer the measured data to the system. Unfortunately, all the commonly available sensors that can be used in the PHC system are not RFID-enabled, and such RFID-enabled devices are too expensive for the low-cost PHC service. The use of more low-cost, RFID-enabled devices would considerably enhance the PHC system and ensure good quality healthcare service.

As of writing this report, the PHC system is working to adopt a software solution to predict an acceptance range by analyzing previous records, called the personal acceptance range [6,19]. If the data cross the acceptance ranges, the system will automatically give a warning, and the health worker will double-check the data to remove any error that may be present. If the patient has insufficient previous data, the system predicts data from subjects of similar age and sex; this is called the group acceptance range. Besides, there is a hypothetical range as a preset of all ages and sex which is called human acceptance range. Once this model is implemented, the PHC system will be able to significantly avoid manual data entry errors and errors attributable to the malfunctioning of medical sensors.

\subsection{Social Impacts}

The PHC service has various social impacts to the service areas. It not only improves the access to good healthcare for rural communities but also engages rural unemployed youths as health workers in the service. Most of the target patients in the rural communities are women who do not have many opportunities to visit distant healthcare facilities; therefore, female health workers are primarily engaged in this home delivery service to ensure the comfort and privacy of female patients. This creates jobs for the rural female population who can find employment in their home village. Thus, as shown in Table 2, the PHC service directly or indirectly contributes to fulfilling various Sustainable Development Goals (SDGs) set by the United Nations [20].

Table 2. PHCs contribution to sustainable development goals

\begin{tabular}{|c|c|c|}
\hline No. & Objective & How does it relate \\
\hline SDG 1 & No poverty & Establishes businesses and employment \\
\hline SGD 2 & Zero hunger & Creating employment opportunity for the poor \\
\hline SDG 3 & Good health and well-being & Population and individual health management \\
\hline SDG 5 & Gender equality & Creating business opportunities for females \\
\hline SDG 8 & Decent work and economic growth & Establishes a business model \\
\hline SDG 9 & Industry, innovation, and infrastructure & $\begin{array}{l}\text { Establishes a collaborative platform where the } \\
\text { device vendors, software solution providers, and } \\
\text { researchers can find their incentives }\end{array}$ \\
\hline SDG 13 & Climate action & Establishes a post-disaster service \\
\hline SDG 17 & Partnerships for the goals & $\begin{array}{l}\text { Creates partnerships between Japan and } \\
\text { Bangladesh and other countries }\end{array}$ \\
\hline
\end{tabular}




\subsection{Geographical Expansion}

As a technologically simple system, the PHC service can be easily replicated and used in any low-resource community. It can be a useful tool for ensuring universal health coverage (UHC) by providing sustainable, doorstep healthcare services in unreached communities of developing countries at an affordable cost. The PHC system has already been implemented in some parts of Cambodia, India, Thailand, and Pakistan with the support of local partners. As of writing this report, this system is being implemented in some other countries and is soon expected to be used in China, Indonesia, and Malaysia.

\section{Conclusions}

The PHC is a modular tele-health system where new modules, such as tele-pathology, tele-eye care, tele-MCH care, and COVID-19 care, have been added over time as per the local needs of the community. With the constant emergence of new requirements, the PHC system will continue to develop new modules and enhance with time, ultimately evolving into a comprehensive tele-health solution.

Given the easy-to-use operation model and portability of the PHC system, it is considered to be an extremely useful tool for use in support shelters during natural disasters and emergencies. Thus, the PHC system aims to adopt a new triage logic for disaster management and support in emergency situations [21].

As discussed above, some technical issues should be addressed. In addition, the PHC system aims to apply the use of artificial intelligence technology for improving the service and lowering the cost. The achievement of these objectives shall be the direction for the next generation of the PHC system.

\section{References}

[1] Nesbitt TS, Marcin JP, Daschbach MM, Cole SL. Perceptions of Local Health Care Quality in 7 Rural Communities with Telemedicine. J Rural Health, 2005, 21(1): 79-85.

[2] A Systematic Review of the Key Indicators for Assessing Telehomecare Cost-Effectiveness. Rojas SV, Gagnon MP. Telemedicine and e-Health November 1, 2008, 14(9): 896-904. doi:10.1089/tmj.2008.0009.

[3] Galiano A, Massaro A; Boussahel B, Barbuzzi D, Tarulli F, Pellicani L, Renna L, Guarini A, De Tullio G, Nardelli G, et al. Improvements in haematology for home health assistance and monitoring by a web based communication system. In Proceedings of the IEEE International Symposium on Medical Measurements and Applications, Benevento, Italy, 15-18 May 2016.

[4] Ahmed A, Rebeiro-Hargrave A, Ghosh PP, Nakashima N, Yasuura H, et al. Portable Health Clinic: A Telehealthcare System for Unreached Communities. In Smart Sensors and Systems. Springer International Publishing, 2015, p. 447-467.

[5] Nohara Y, Kai E, Ghosh P, Kitsuregawa M, Nakashima N, et al. Health Checkup and Telemedical Intervention Program for Preventive Medicine in Developing Countries: Verification Study. Journal of Medical Internet Research, 2015, 17, 1.

[6] Ahmed A, Hasan M, Sampa MB, Hossein KM, Nohara Y, Nakashima N. Chapter 7: Portable health clinic: concept, design, implementation and challenges. Book: Mobile Technologies for Delivering Healthcare in Remote, Rural or Developing Regions, Published by IET, London, UK, 2020, p. 105-121.

[7] Islam R, Nohara Y, Rahman JU, Sultana N, Ahmed A, Nakashima N. Portable health clinic: An advanced tele-healthcare system for unreached communities: MEDINFO 2019: Health \& Wellbeing e-Network for All, Volume 264, 2019, p. 416-419.

[8] Yokota F, Ahmed A, Kikuchi K, Nishikitani M, Islam R, Nakashima N. Diabetes, obesity, and hypertension in Bheramara Kushtia District, Bangladesh - Results from portable health clinic data, 20132016. Proceeding of Social Business Academia Conference, 2016. 
[9] Bombard Y, Baker GR, Orlando E, et al. Engaging patients to improve quality of care: A systematic review. Implement Sci. 2018;13(1):98. Published 2018 Jul 26.

[10] Bae WK, Kwon J, Lee HW, et al. Feasibility and accessibility of electronic patient-reported outcome measures using a smartphone during routine chemotherapy: A pilot study. Support Care Cancer. 2018;26(11):3721-3728.

[11] Nakashima N, Noda M, Ueki K, et al. Recommended configuration for personal health record by standardized data item sets for diabetes mellitus and associated chronic diseases: a report from collaborative initiative by six Japanese associations. Diabetology International, 2019, 10(2):85-92.

[12] Islam R, Kikuchi K, Sato Y, Izukura R, Nishikitani M. Chapter 8: Modular expansion of PHC in Bangladesh. Book: Mobile Technologies for Delivering Healthcare in Remote, Rural or Developing Regions, Published by IET, London, UK, 2020, p. 123-143.

[13] Islam R, Rahman MJ, Ahmed A, Nakashima N. GramHealth: Tele-Pathology System for PHC Telemedicine Service. Proceeding of the 4th Digital Pathology CONGRESS: ASIA, 2018.

[14] Kikuchi K, Sato Y, Izukura R, Nishikitani M, Kato K, Morokuma S, et al. Portable health clinic for sustainable care of mothers and newborns in rural Bangladesh. Conference Proceeding of Asia-Pacific Association for Medical Informatics. 2020.

[15] WHO. Pregnancy, childbirth, postpartum and newborn care: A guide for essential practices. Geneva, Switzerland: WHO; 2003.

[16] Sampa MB, Hoque MR, Islam R, Nishikitani M, Nakashima N, et al. Redesigning portable health clinic platform as a remote healthcare system to tackle COVID-19 pandemic situation in unreached communities. International Journal of Environmental Research and Public Health. 17, 4709, 2020.

[17] Jacobs B. Electronic medical record, error detection, and error reduction: A pediatric critical care perspective. Pediatr. Crit. Care Med. 2007, 8, 17-20.

[18] Goldberg SI, Niemierko A, Turchin A. Analysis of data errors in clinical research databases. AMIA Annu. Symp. Proc. 2008, 2008, 242-246.

[19] Hasan M, Yokota Y, Islam R, Hisazumi K, Fukuda A, Ahmed A. A predictive model for height tracking in an adult male population in Bangladesh to reduce input errors. International Journal of Environmental Research and Public Health 2020, 17, 1806.

[20] SDG Tracker, Bangladesh. Accessed December 2020 from: https://www.sdg.gov.bd/page/indicatorwise $/ 5 / 427 / 2 / 5 \# 1$

[21] Hu M, Sugimoto M, Hargrave AR, Moriyama M, Shimizu S, et al. Mobile healthcare system for health checkups and telemedicine in post-disaster situations. Stud Health Technol Inform 2015, 216:7983. 